\title{
Association between heparin-binding hemagglutinin and tuberculosis
}

\author{
${ }^{*}$ Feifei Pu ${ }^{\mathrm{A}, \mathrm{B}},{ }^{*}$ Jing Feng ${ }^{\mathrm{D}, \mathrm{E}}$, Ping Xia ${ }^{\mathrm{E}, \mathrm{F}}$ \\ and Technology, China \\ A - research concept and design; $\mathrm{B}$ - collection and/or assembly of data; $\mathrm{C}$ - data analysis and interpretation; \\ $D$ - writing the article; $E$ - critical revision of the article; $F$ - final approval of the article
}

Department of Orthopedics, Wuhan No. 1 Hospital (Wuhan Integrated TCM \& Western Medicine Hospital), Tongji Medical College, Huazhong University of Science

\section{Address for correspondence \\ Ping Xia \\ E-mail:xiapingfm@163.com}

\section{Funding sources}

This work was supported by a research project of the Hubei Health and Family Planning Commission (grant No. WJ2017Z022), a research project of the Hubei Science and Technology Department (grant No. 2016CFB666), a research project of the Wu Jieping Medical Foundation (grant No. 320.6750.17566), a research project of the Wuhan Health and Family Planning Commission (grants No. WZ18005 and No. WX18Z20), and a research project of Wuhan No. 1 Hospital (Wuhan Integrated TCM \& Western Medicine Hospital) (grant No. 2017Y01).

Conflict of interest

None declared

* Feifei Pu and Jing Feng have made equal contributions to this work.

Received on December 24, 2018

Reviewed on April 24, 2020

Accepted on April 24, 2020

Published online on July 31, 2020

\begin{abstract}
The current global burden of tuberculosis (TB) is one of the greatest challenges to public health, particularly in developing countries, and thus effective diagnostic methods and treatment options for TB remain a central topic in basic and clinical research. Heparin-binding hemagglutinin (HBHA)-specific immune responses have been linked to protection against TB. The binding of HBHA-coated beads to epithelial and endothelial cell layers may trigger transcytosis of the beads, which is the basis for extrapulmonary dissemination. In addition, HBHA has been confirmed as a potential diagnostic marker for TB, and it is important in developing new TB vaccines and anti-TB drugs. Recently, basic research on HBHA has been intensified. The HBHA application in the field of prevention and treatment should be further explored. In addition, the existing research achievements have shown its broad application prospects. Currently, there are no relevant specialized products, and research should be accelerated. These products may contribute to the application of HBHA in the diagnosis, prevention and treatment of TB.
\end{abstract}

Key words: heparin-binding hemagglutinin, tuberculosis, diagnosis, treatment

Cite as

Pu F, Feng J, Xia P. Association between heparin-binding hemagglutinin and tuberculosis. Adv Clin Exp Med. 2020;29(7):893-897. doi:10.17219/acem/121011

DOI

10.17219/acem/121011

\section{Copyright}

Copyright by Author(s)

This is an article distributed under the terms of the

Creative Commons Attribution 3.0 Unported (CC BY 3.0)

(https://creativecommons.org/licenses/by/3.0/) 


\section{Introduction}

Tuberculosis (TB), an infectious disease caused by $M y c o$ bacterium tuberculosis (Mtb), remains a global health problem, with an estimated 10.4 million incident cases of active TB (aTB) in $2015 .^{1}$ It is a major infectious disease that kills almost two million people every year, mostly in developing countries, where it poses a major health, social and economic burden. ${ }^{2}$ The development of improved, clinically sensitive, rapid, and economical diagnostic tests may provide a powerful tool to better control the epidemic. Recently, polymerase chain reaction (PCR)-based methods and automatic culture systems have been made available, and these methods are in extensive regular use in laboratories in developed countries. ${ }^{3}$ However, these diagnostic systems are not suitable for field use. The idea to develop a test for TB diagnosis through a serological assay has been pursued for many decades, but the results so far have been poor. Many of the antigens which have been tested did not possess adequate sensitivity or specificity, and these assays could not properly distinguish between individuals vaccinated with the Mycobacterium bovis bacillus Calmette-Guérin (BCG) vaccine or testing positive for purified protein derivative (PPD) and those with aTB. ${ }^{4}$

Recently, it has been demonstrated that several mycobacterial proteins undergo a process of post-translational modification in mycobacteria, providing important immunological properties. Among these proteins is heparin-binding hemagglutinin (HBHA), which is methylated in lysine residues present at the C-terminus. ${ }^{5,6}$ Since the recombinant proteins produced in Escherichia coli cannot be properly processed, the use of these antigens in serological assays is precluded by the cumbersome procedures required to purify the native antigens. Recently, a rapid and effective system for the purification of methylated HBHA (mHBHA) has been developed, and the use of these proteins in such assays is now feasible. ${ }^{7}$ The HBHA plays an important role during infection, especially during extrapulmonary dissemination to other systems in the human host. It has also been investigated as a potential diagnostic tool for latent $\mathrm{TB}$, as a possible vaccine and as a booster of BCG immunity, probably by inducing cytokines that may contribute to the generation of $\mathrm{T}$ helper 1 (Th1) effector memory lymphocytes. The HBHA is known to bind to heparin.

The HBHA of Mtb, a dissemination factor that has been identified as an immunodominant antigen able to induce interferon gamma (IFN $\gamma$ ), is associated with protection against TB. ${ }^{8}$ This surface protein interacts with nonphagocytic cells, attaches to sulfated glycoconjugates, and has the ability to agglutinate red blood cells. ${ }^{9,10}$ The specific methylation of the antigen has been shown to be important in mounting an immune response against this antigen, and it has been suggested that HBHA can be used in IFN $\gamma$ release assays (IGRAs) to diagnose Mtb infection or to discriminate between the active and latent forms of the disease. Current preclinical strategies involve administering the HBHA vaccine as a booster following vaccination with BCG. ${ }^{11}$

\section{Material and methods}

A systematic search of electronic databases, including PubMed, EMBASE and Google Scholar, was performed in order to identify relevant articles (published until November 21, 2018) characterizing the role of HBHA in TB. To maximize the number of eligible articles, the following keywords were used in combination: [HEPARIN-BINDING HEMAGGLUTININ], [HBHA] and [TUBERCULOSIS]. Additional studies were sought from the references of all the articles retrieved; only studies examining HBHA in TB were included, while non-English language studies and review articles were excluded.

Two independent reviewers screened all the titles and abstracts, and disagreements related to inclusion or exclusion of a particular study were resolved through consensus. Once the study was selected for inclusion based on its title and abstract, the full text was further screened by 2 independent reviewers. A total of 88 articles were identified.

\section{Discussion}

\section{Biochemical characteristics of HBHA}

The encoding gene of hbha is approx. $600 \mathrm{bp}$ long, and the corresponding protein is composed of 199 amino acids. The molecular mass is about 28,000 Da. The HBHA contains 3 domains: a transmembrane domain composed of 18 amino acid residues at the $\mathrm{N}$-terminus, a helical region containing 81 amino acid residues of curly helical type, and it can be modified by methylation after translation into the C-terminal junction. ${ }^{12,13}$ The $\mathrm{N}$-terminal transmembrane region was inserted into the surface membrane to fix HBHA to the lipid membrane of the surface of the bacteria, thereby enhancing virulence, while the $C$ terminal domain plays an important role in mediating combination and sulfating the polysaccharide receptor., ${ }^{9,10}$

The HBHA can form a homodimer or polymer. The molecular weight of HBHA was measured using force microscopy. ${ }^{14}$ Peaks represent HBHA forming dimers or polymers. At the same time, it is also found that similar effects exist in viable bacteria that produce HBHA. This phenomenon was not found in the hbha gene deletion mutant. Combined with the fact that HBHA mediates bacterial agglutination, it is speculated that a dimer or a polymer is formed through the interaction of the source HBHA and body agglutination. Methylation prevents the hydrolysis of HBHA protein. ${ }^{15}$ 


\section{Expression of HBHA in different carriers}

Although it is generally accepted that the interaction of Mtb with alveolar macrophages is a key step in the pathogenesis of TB, interactions with other cell types, especially epithelial cells, may also be important. ${ }^{16}$ In this study, we characterized the molecular properties of mycobacterial HBHA, a protein that functions as an adhesin for epithelial cells. ${ }^{17}$ The gene was cloned from Mtb and M. bovis BCG, and the sequence was found to be identical between the 2 species. The calculated result was smaller than the finding from SDS/PAGE analysis; this difference may be due to the Lys/Pro-rich repeats of the $\mathrm{C}$-terminal end of the protein and to a putative carbohydrate moiety. Glycosylation of HBHA appears to protect the protein from proteolytic degradation, which results in the removal of the $\mathrm{C}$-terminal Lys/Pro-rich region responsible for binding HBHA to sulfated carbohydrates. ${ }^{18}$ Evidence suggests that glycosylation is also important for HBHA-mediated hemagglutination and for certain immunological properties of the protein. Finally, the absence of a signal peptide in the coding region of HBHA raises the possibility that this protein is not secreted through the general secretory pathway.

The Mtb and M. bovis BCG HBHA is required for extrapulmonary dissemination, and laminin-binding protein (LBP) is involved in cytoadherence through laminin recognition. ${ }^{19,20}$ These adhesins bear post-translational modifications that are not present when the proteins are produced in a recombinant (r) form in E. coli. Mass spectrometry analysis of HBHA reveals that the post-translational modifications are borne by the C-terminal moiety, which comprises the heparin-binding domain consisting of repeated lysine-rich motifs. Amino acid sequencing showed that these modifications consist of mono- and dimethyllysines within these motifs. The methyllysinecontaining repeats were recognized by $\mathrm{mAb} 4057 \mathrm{D} 2$ and were also detected in LBP, which is also recognized by $\mathrm{mAb}$ 4057D2. This antibody does not recognize the recombinant forms of these proteins. However, when recombinant HBHA (rHBHA) and rLBP were subjected to $\mathrm{NaOH}$ and formalin treatment to induce lysine methylation, reactivity with mAb 4057D2 was recovered. Methylated rHBHA displayed enhanced resistance to proteolysis compared with unmethylated rHBHA, as previously observed for native HBHA. S-adenosylmethionine-dependent HBHA methyltransferase activity was detected in the cell-wall fractions of $M$. bovis BCG and of Mycobacterium smegma$t i s$, a species that produces LBP but naturally lacks HBHA, suggesting that the same enzyme methylates both LBP and HBHA. This hypothesis was confirmed by the fact that HBHA produced by recombinant $M$. smegmatis was also methylated. These results show that mycobacteria use enzymatic methylation of lysines to ensure greater stability of their adhesins.
A recombinant $M$. smegmatis strain that overexpressed HBHA under the control of a strong furA promoter was constructed to discriminate IGRAs between aTB and latent tuberculosis infection (LTBI). The methylated activity of the purified protein was verified using hybridization with anti-methylated Lys antibody, and mHBHA was further evaluated for antigen-specific IFN $\gamma$ responses in a BCG-vaccinated Chinese population. Taken together, evaluation of the immune response to mHBHA can discriminate between healthy LTBI cases and aTB patients.

\section{Infection and pathogenesis of HBHA and $M$. tuberculosis}

By virtue of their position at the crossroads between the innate and adaptive immune response, macrophages play an essential role in the control of bacterial infections. Paradoxically, macrophages serve as the natural habitat of Mtb. The Mtb subverts the macrophage's mechanisms of intracellular killing and antigen presentation, ultimately leading to the development of TB. The HBHA, a virulence factor involved in extrapulmonary dissemination and a strong diagnostic antigen for TB, is both surfaceassociated and secreted. Disruption of the hbha gene led to the restoration of impairment in infected macrophages, resulting in reduced apoptosis. Taken together, the data suggests that HBHA may act as a strong pathogenic factor to cause apoptosis of professional phagocytes infected with Mtb. ${ }^{21} \mathrm{~A}$ recent study suggested that HBHA induces cytosolic $[\mathrm{Ca}(2+)] \mathrm{i}$, which influences the generation of reactive oxygen species (ROS) associated with the production of pro-inflammatory cytokines. These concerted and complex cellular responses induce endoplasmic reticulum stress-associated apoptosis during HBHA stimulation in macrophages. These results indicate that the endoplasmic reticulum stress pathway contributes significantly to HBHA-induced apoptosis during mycobacterial infection. $^{22}$

Importantly, in vitro studies have demonstrated that the Mtb $h b h a$ and esxA gene products HBHA and ESAT6 directly or indirectly influence alveolar epithelial cell survival. ${ }^{23}$ In vitro assays with the recombinant histone-like protein and HBHA, which are considered to be major mycobacterial adhesins, confirmed their function of promoting bacterial attachment to epithelial cells. In addition, these experiments suggested that the airway epithelium may act as a reservoir and/or portal of entry for Mycobacterium leprae in humans. ${ }^{24} \mathrm{~A}$ previous study used immunoblotting to examine the humoral immune responses of aTB patients against triton-soluble proteins extracted from Mtb. Of interest, the pooled sera from TB patients that contained anti-HBHA IgM antibodies neutralized the entry of Mtb into epithelial cells. These findings suggest that IgM antibodies against HBHA may help protect against extrapulmonary dissemination. ${ }^{25}$ 


\section{Detection of HBHA in tuberculosis immunology}

In recent years, T-cell-based IGRAs have been developed for immunodiagnosis of Mtb infection. Currently, these assays do not discriminate between disease and latency. Therefore, more promising antigens and diagnostic tools are continuously being searched for TB immunodiagnostics. The HBHA is a surface protein of Mtb, which promotes bacterial aggregation and adhesion to non-phagocytic cells. It has been previously assumed that the native, methylated form of this protein would be a promising antigen to discriminate between latent and active infection. A pilot investigation was performed to study the humoral and T-cell-mediated immunological responses to recombinant HBHA produced in M. smegmatis and to synthetic peptides in patients with recent or past TB, with atypical mycobacteriosis, and in healthy, vaccinated individuals. T-cell reactivity to $\mathrm{HBHA}$ was compared to the respective reactivity toward PPD and 2 surface-secreted proteins, i.e., early secretory antigen target-6 (ESAT-6) and culture filtrate protein-10 (CFP-10). ${ }^{26}$ The preliminary results indicate that methylated $\mathrm{rHBHA}$ induced a strong T-cell-mediated immune response and the production of IgG and IgM-class antibodies in all patient groups, most surprisingly in young Finnish vaccinees. They observed a positive correlation between the reactivity to HBHA and non-specific PPD among the entire study population. As expected, ESAT- 6 and CFP-10 were the most powerful antigens to distinguish between disease and immunity caused by vaccination. ${ }^{27}$ On the basis of this exploratory investigation, we raise concerns that in countries like Finland, where BCG vaccination was routinely used, $\mathrm{BBHA}$ may not be sufficient for diagnostics because of the inability to explicitly discriminate between TB infection and immunoreactivity caused by previous BCG vaccination. ${ }^{28}$

The IGRAs based on region of difference 1 (RD1) antigens have improved the diagnosis of Mtb infection. However, IGRAs of these antigens cannot distinguish between aTB and LTBI. The Mtb HBHA induces relatively high IFN $\gamma$ responses in LTBI individuals and low responses in aTB patients, but purification of the native methylated HBHA from Mtb cultures for immunological tests is complex and time-consuming. The study showed that T-cell responses against $\mathrm{mHBHA}$ were always lower in aTB patients than in LTBI individuals, regardless of the site of infection or the results of bacteriological tests. This allowed for a high discrimination between these 2 groups of Mtbinfected individuals, even in the BCG-vaccinated and highTB-incidence setting of China. Additionally, the combination of mHBHA and RD 1 antigens in an IGRA enhanced the diagnostic efficacy for aTB cases. Taken together, inclusion of the immune response to $\mathrm{mHBHA}$ can differentiate between healthy LTBI cases and aTB patients. ${ }^{15}$

In a recent study, 165 outpatients and 133 healthy volunteers were included in an investigation of the role of HBHA in TB diagnosis, including serodiagnostic tests and IGRAs. None of the healthy volunteers had been BCG-vaccinated, including 73 individuals with PPD (-) and 60 with PPD (+) (i.e., P-B- and P+B-). Of all 165 outpatients, 77 had pulmonary tuberculosis (PTB) and 88 had extrapulmonary tuberculosis (EPTB). The HBHA protein was used for serodiagnostic tests and IGRAs in peripheral blood mononuclear cells. They found that HBHA serodiagnostic tests with IGRAs had a limited potential for use as auxiliary tools for the differential diagnosis of PTB and EPTB, because both methods showed low sensitivity and specificity. ${ }^{29}$

\section{Conclusions and future prospects}

Tuberculosis is one of the greatest challenges to public health, particularly in developing countries, and thus effective diagnostic methods and treatment options for TB remain a central topic in basic and clinical research. The HBHA-specific immune responses have been linked to protection against TB. Lately, the investigation of HBHA in basic research has been intensified. The HBHA application in the field of prevention and treatment should be further explored. In addition, recent findings have shown its broad application prospects. Currently, there are no relevant specialized products, and research should be accelerated. These products may contribute to the application of $\mathrm{HBHA}$ in the diagnosis, prevention and treatment of TB.

\section{ORCID iDs}

Feifei Pu (1) https://orcid.org/0000-0002-9174-8780

Jing Feng (1) https://orcid.org/0000-0001-9895-6534

Ping Xia (1) https://orcid.org/0000-0002-5544-3082

\section{References}

1. Wang $Y$, Wang $Q$, Zhu R, et al. Trends of spinal tuberculosis research (1994-2015): A bibliometric study. Medicine (Baltimore). 2016;95(38): e4923.

2. Kumar K. Spinal tuberculosis: Natural history of disease, classifications and principles of management with historical perspective. Eur J Orthop Surg Traumatol. 2016;26(6):551-558.

3. Chen $\mathrm{CH}$, Chen YM, Lee CW, Chang YJ, Cheng CY, Hung JK. Early diagnosis of spinal tuberculosis. J Formos Med Assoc. 2016;115(10): 825-836.

4. Nagashima H, Tanishima S, Tanida A. Diagnosis and management of spinal infections. J Orthop Sci. 2018;23(1):8-13.

5. De Maio F, Squeglia F, Goletti D, Delogu G. The mycobacterial HBHA protein: A promising biomarker for tuberculosis. Curr Med Chem. 2018;26(11):2051-2060. doi:10.2174/0929867325666181029165805

6. Raze D, Verwaerde C, Deloison G, et al. Heparin-binding hemagglutinin adhesin (HBHA) is involved in intracytosolic lipid inclusions formation in Mycobacteria. Front Microbiol. 2018;9:2258.

7. Sali M, Buonsenso D, D'Alfonso P, et al. Combined use of Quantiferon and HBHA-based IGRA supports tuberculosis diagnosis and therapy management in children. J Infect. 2018;77(6):526-533.

8. Loxton AG, Black GF, Stanley K, WalzI G. Heparin-binding hemagglutinin induces IFN- $\gamma(+)$ IL-2(+) IL-17(+) multifunctional CD4(+) T cells during latent but not active tuberculosis disease. Clin Vaccine Immunol. 2012; 19(5):746-751.

9. Lanfranconi MP, Alvarez HM. Functional divergence of HBHA from Mycobacterium tuberculosis and its evolutionary relationship with TadA from Rhodococcus opacus. Biochimie. 2016;127:241-248. 
10. Huang TY, Irene D, Zulueta MM, et al. Structure of the complex between a heparan sulfate octasaccharide and mycobacterial heparin-binding hemagglutinin. Angew Chem Int Ed Engl. 2017;56(15):4192-4196.

11. Segura-Cerda CA, Aceves-Sánchez MJ, Marquina-Castillo B, et al. Immune response elicited by two rBCG strains devoid of genes involved in c-di-GMP metabolism affect protection versus challenge with M. tuberculosis strains of different virulence. Vaccine. 2018;36(16): 2069-2078.

12. Delogu G, Brennan MJ. Functional domains present in the mycobacterial hemagglutinin, HBHA. J Bacteriol. 1999;181(24):7464-7469.

13. Biet $F$, de Melo Marques MA, Grayon M, et al. Mycobacterium smegmatis produces an HBHA homologue which is not involved in epithelial adherence. Microbes Infect. 2007;9(2):175-182.

14. Esposito C, Cantisani M, D'Auria G, et al. Mapping key interactions in the dimerization process of HBHA from Mycobacterium tuberculosis, insights into bacterial agglutination. FEBS Lett. 2012;586(6):659-667.

15. Wen HL, Li CL, Li G, et al. Involvement of methylated HBHA expressed from Mycobacterium smegmatis in an IFN- $\gamma$ release assay to aid discrimination between latent infection and active tuberculosis in BCG-vaccinated populations. Eur J Clin Microbiol Infect Dis. 2017;36(8):1415-1423.

16. Raze D, Verwaerde C, Deloison G, Berisio R. A structural overview of mycobacterial adhesins: Key biomarkers for diagnostics and therapeutics. Protein Sci. 2018;27(2):369-380.

17. Silva CA, Danelishvili L, McNamara M, et al. Interaction of Mycobacterium leprae with human airway epithelial cells: Adherence, entry, survival, and identification of potential adhesins by surface proteome analysis. Infect Immun. 2013;81(7):2645-2659.

18. Lefrancois LH, Bodier CC, Lecher S, et al. Purification of native HBHA from Mycobacterium avium subsp. paratuberculosis. BMC Res Notes. 2013;6:55.

19. Pethe $K$, Bifani $P$, Drobecq $H$, et al. Mycobacterial heparin-binding hemagglutinin and laminin-binding protein share antigenic methyllysines that confer resistance to proteolysis. Proc Natl Acad Sci U SA. 2002;99(16):10759-10764.

20. Pethe K, Puech V, Daffé M, et al. Mycobacterium smegmatis lamininbinding glycoprotein shares epitopes with Mycobacterium tuberculosis heparin-binding haemagglutinin. Mol Microbiol. 2001;39(1):89-99.
21. Sohn H, Kim JS, Shin SJ, et al. Targeting of Mycobacterium tuberculosis heparin-binding hemagglutinin to mitochondria in macrophages. PLoS Pathog. 2011;7(12):e1002435.

22. Choi JA, Lim YJ, Cho SN, et al. Mycobacterial HBHA induces endoplasmic reticulum stress-mediated apoptosis through the generation of reactive oxygen species and cytosolic $\mathrm{Ca} 2+$ in murine macrophage RAW 264.7 cells. Cell Death Dis. 2013;4(12):e957.

23. Pavlicek RL, Fine-Coulson K, Gupta T, et al. Rv 3351c, a Mycobacterium tuberculosis gene that affects bacterial growth and alveolar epithelial cell viability. Can J Microbiol. 2015;61(12):938-947.

24. Silva CA, Danelishvili L, McNamara M, et al. Interaction of Mycobacterium leprae with human airway epithelial cells: Adherence, entry, survival, and identification of potential adhesins by surface proteome analysis. Infect Immun. 2013;81(7):2645-2659.

25. Shin AR, Lee KS, Lee JS, et al. Mycobacterium tuberculosis HBHA protein reacts strongly with the serum immunoglobulin $\mathrm{M}$ of tuberculosis patients. Clin Vaccine Immunol. 2006;13(8):869-875.

26. Dirix V, Schepers K, Massinga-Loembe M, et al. Added value of longterm cytokine release assays to detect Mycobacterium tuberculosis infection in HIV-infected subjects in Uganda. J Acquir Immune Defic Syndr. 2016;72(3):344-352.

27. Hutchinson P, Barkham TM, Tang W, Kemeny DM, Bin-Eng Chee C, Wang YT. Measurement of phenotype and absolute number of circulating heparin-binding hemagglutinin, ESAT- 6 and CFP-10, and purified protein derivative antigen-specific CD4 T cells can discriminate active from latent tuberculosis infection. Clin Vaccine Immunol. 2015;22(2):200-212.

28. Savolainen L, Pusa L, Kim HJ, Sillanpää H, Seppälä I, Tuuminen T. Pilot study of diagnostic potential of the Mycobacterium tuberculosis recombinant $\mathrm{HBHA}$ protein in a vaccinated population in Finland. PLoS One. 2008;(9):e3272.

29. Sun Z, Nie L, Zhang X, Li Y, Li C. Mycobacterial heparin-binding haemagglutinin adhesion-induced interferon \& antibody for detection of tuberculosis. Indian J Med Res. 2011;133(4):421-425. 